\title{
Evidence for quantum chaos in the plasma phase of QCD
}

\author{
R. Pullirsch ${ }^{\mathrm{a}}$, K. Rabitsch ${ }^{\mathrm{a}}$, T. Wettig ${ }^{\mathrm{b}}$, and H. Markum ${ }^{\mathrm{a}}$ \\ anstitut für Kernphysik, Technische Universität Wien, A-1040 Wien, Austria \\ ${ }^{\mathrm{b}}$ Institut für Theoretische Physik, Technische Universität München, D-85747 \\ Garching, Germany
}

\begin{abstract}
We investigate the eigenvalue spectrum of the staggered Dirac matrix in $\mathrm{SU}(3)$ gauge theory and in full QCD on a $6^{3} \times 4$ lattice. As a measure of the fluctuation properties of the eigenvalues, we study the nearest-neighbor spacing distribution $P(s)$ for various values of $\beta$ both in the confinement and in the deconfinement phase. In both phases except far into the deconfinement region, the lattice data agree with the Wigner surmise of random-matrix theory which is indicative of quantum chaos. We do not find signs of a transition to Poisson regularity at the deconfinement phase transition.
\end{abstract}

\section{Introduction}

The properties of the eigenvalues of the Dirac operator are of great importance for the understanding of certain features of QCD. For example, the accumulation of small eigenvalues is, via the Banks-Casher formula [1], related to the spontaneous breaking of chiral symmetry. Recently, the fluctuation properties of the eigenvalues in the bulk of the spectrum have also attracted attention. It was shown in Ref. [2] that on the scale of the mean level spacing they are described by random-matrix theory (RMT). In particular, the nearest-neighbor spacing distribution $P(s)$, i.e., the distribution of spacings $s$ between adjacent eigenvalues on the unfolded scale (see below), agrees with the Wigner surmise of RMT. According to the so-called Bohigas-conjecture [3], quantum systems whose classical counterparts are chaotic have a nearest-neighbor spacing distribution given by RMT whereas systems whose classical counterparts are integrable obey a Poisson distribution, $P(s)=e^{-s}$. Therefore, the specific form of $P(s)$ is often taken as a criterion for the presence or absence of "quantum chaos". It should be noted, however, that there is no universally accepted proof of the Bohigas-conjecture yet. The field of quantum chaos is 
still developing, and there are many open theoretical problems. For reviews on this subject we refer to Ref. [4].

A transition in $P(s)$ from Wigner to Poisson behavior is observed at the localization transition in disordered mesoscopic systems [5]. In Ref. [2], the question was raised if there is such a transition from chaotic to regular behavior in the case of the lattice Dirac operator, and the present study serves to investigate this question. For a preliminary account of results for quenched QCD, see Ref. [6]. Recently, a Wigner to Poisson transition was also studied in the context of a spatially homogeneous Yang-Mills-Higgs system [7]. The question if chaos in an $N$-component $\varphi^{4}$-theory in the presence of an external field survives quantization was investigated in Ref. [8]. An analytical treatment of the transition between Wigner and Poisson behavior can be found in Ref. [9].

In RMT, one has to distinguish between several universality classes which are determined by the symmetries of the system. For the case of the QCD Dirac operator, this classification was done in Ref. [10]. Depending on the number of colors and the representation of the quarks, the Dirac operator is described by one of the three chiral ensembles of RMT. As far as the fluctuation properties in the bulk of the spectrum are concerned, the predictions of the chiral ensembles are identical to those of the ordinary ensembles [11]. In Ref. [2], the Dirac matrix was studied in $\mathrm{SU}(2)$ using both staggered and Wilson fermions which correspond to the symplectic and orthogonal ensemble, respectively. Here, we study $\mathrm{SU}(3)$ with staggered fermions which corresponds to the chiral unitary ensemble. We thus cover the last remaining symmetry class in the confined phase. Although the unitary ensemble is, from a mathematical point of view, the simplest one, the RMT result for the nearest-neighbor spacing distribution is still rather complicated. It can be expressed in terms of so-called prolate spheroidal functions, see Ref. [12] where $P(s)$ has also been tabulated. A very good approximation to $P(s)$ is provided by

$$
P(s)=\frac{32}{\pi^{2}} s^{2} e^{-\frac{4}{\pi} s^{2}} .
$$

This is the Wigner surmise for the unitary ensemble.

\section{Eigenvalue analysis}

We generated gauge field configurations using the standard Wilson plaquette action for SU(3) with and without dynamical fermions in the Kogut-Susskind prescription. We have worked on a $6^{3} \times 4$ lattice with various values of the inverse gauge coupling $\beta=6 / g^{2}$ both in the confinement and deconfinement phase. The boundary conditions were periodic for the gluons and periodic in 
space and anti-periodic in Euclidean time for the fermions. We typically produced 10 independent equilibrium configurations (separated by 1000 sweeps) for each $\beta$. Because of the spectral ergodicity property of RMT one can replace ensemble averages by spectral averages if one is only interested in bulk properties, and thus one does not need a large number of independent configurations to compute $P(s)$.

By definition, the Dirac operator $\not D=\not \partial+i g A$ from which the spectrum is calculated does not contain the quark mass term. The operator is antihermitian so that all eigenvalues are imaginary. For convenience, we denote them by $i \lambda_{n}$ and refer to the $\lambda_{n}$ as the eigenvalues in the following. Because of $\left\{\not D, \gamma_{5}\right\}=0$ the $\lambda_{n}$ occur in pairs of opposite sign. All spectra were checked against the analytical sum rules

$$
\sum_{n} \lambda_{n}=0 \quad \text { and } \quad \sum_{\lambda_{n}>0} \lambda_{n}^{2}=3 V
$$

where $\mathrm{V}$ is the lattice volume.

To construct the nearest-neighbor spacing distribution $P(s)$ from the eigenvalues, one first has to "unfold" the spectra. This procedure is a local rescaling of the energy scale so that the mean level spacing is equal to unity on the unfolded scale. One first defines the staircase function $N(E)$ to be the number of eigenvalues with $\lambda \leq E$. This staircase function has an average part and a fluctuating part, $N(E)=N_{\mathrm{av}}(E)+N_{\mathrm{fl}}(E)$. The average part is extracted by fitting $N(E)$ to a smooth curve, e.g., to a low-order polynomial. One then defines the unfolded energies to be $x_{n}=N_{\mathrm{av}}\left(E_{n}\right)$. As a consequence, the sequence $\left\{x_{n}\right\}$ has mean level spacing equal to unity. Ensemble and spectral averages are only meaningful after unfolding.

\section{Results and Discussion}

We begin the discussion of our results with the pure gluonic case. Figure 1 shows the staircase function $N(E)$ which in the continuum is given by $N(E)=$ $\int_{0}^{E} \rho(\lambda) d \lambda$, where $\rho(\lambda)$ is the spectral density. Note that $\rho(\lambda)$ cannot be obtained universally from a random-matrix model. The staircase function is not very sensitive to the value of $\beta$, neither in the confinement nor in the deconfinement regime. However, across the phase transition $N(E)$ is diminished for small $E$ reflecting the suppression of small eigenvalues.

Figure 2 shows the nearest-neighbor spacing distribution $P(s)$ corresponding to the parameters of Fig. 1, compared with the RMT prediction. In the confinement phase, we find the expected agreement of $P(s)$ with the Wigner surmise 


\section{Confinement}

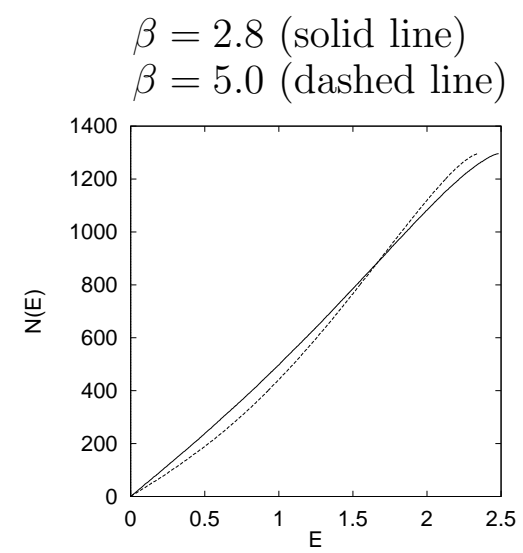

Deconfinement

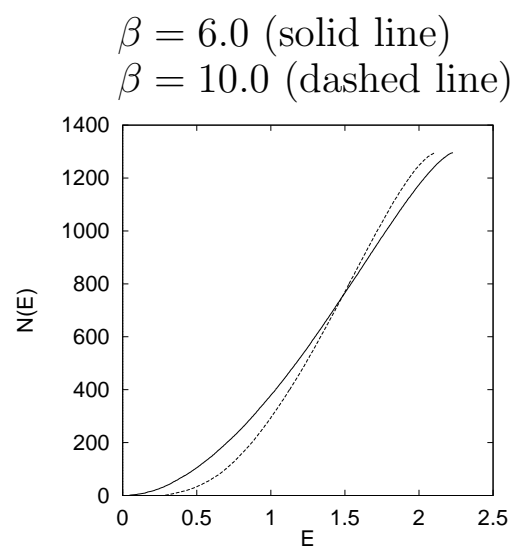

Fig. 1. The staircase function $N(E)$ representing the number of positive eigenvalues $\leq E$ for typical configurations on a $6^{3} \times 4$ lattice in pure $\mathrm{SU}(3)$.

of Eq. (1). In the deconfinement phase, we still observe agreement with the RMT result up to $\beta=10.0$ (we have also plotted the Poisson distribution for comparison).

We continue with the case of full QCD with $N_{f}=3$ degenerate flavors of staggered quarks with mass $m a=0.1$ and $m a=0.05$, respectively. The staircase function $N(E)$ in Fig. 3 shows practically no dependence on the masses considered.

Figure 4 compares the nearest-neighbor spacing distribution $P(s)$ of full QCD with the RMT result. No mass dependence is visible. Again, in the confinement as well as in the deconfinement phase we still observe agreement with the RMT result up to $\beta=8.0$ (not shown). The observation that $P(s)$ is not influenced by the presence of dynamical quarks could have been expected from the results of Ref. [11]. Those calculations, however, only apply to the case of massless dynamical quarks. Our results, as those of Ref. [2], strongly indicate that massive dynamical quarks do not affect $P(s)$ either.

No signs for a transition to Poisson regularity are found. Thus, the deconfinement phase transition does not seem to coincide with a transition in the spacing distribution. For very large values of $\beta$ far into the deconfinement region (not shown), the eigenvalues start to approach the degenerate eigenvalues of the free theory, given by $\lambda^{2}=\sum_{\mu=1}^{4} \sin ^{2}\left(2 \pi n_{\mu} / L_{\mu}\right) / a^{2}$, where $a$ is the lattice constant, $L_{\mu}$ is the number of lattice sites in the $\mu$-direction, and $n_{\mu}=0, \ldots, L_{\mu}-1$. In this case, the nearest-neighbor spacing distribution is neither Wigner nor Poisson. However, it is possible to lift the degeneracies of the free eigenvalues using an asymmetric lattice where $L_{x}, L_{y}$, etc. are relative primes [13]. For large lattices, the nearest-neighbor spacing distribution of the non-degenerate free eigenvalues is then given by the Poisson distribu- 


\section{Confinement}
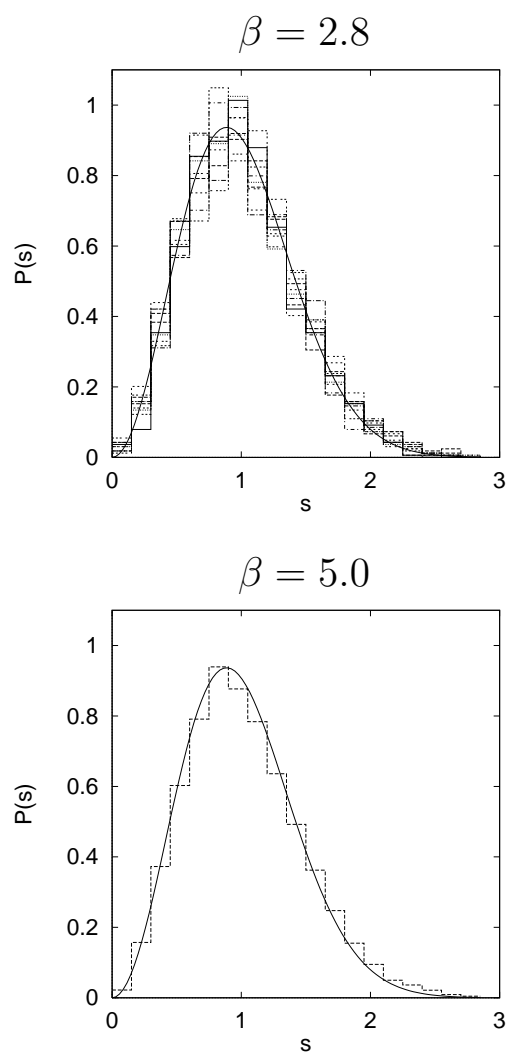

Deconfinement
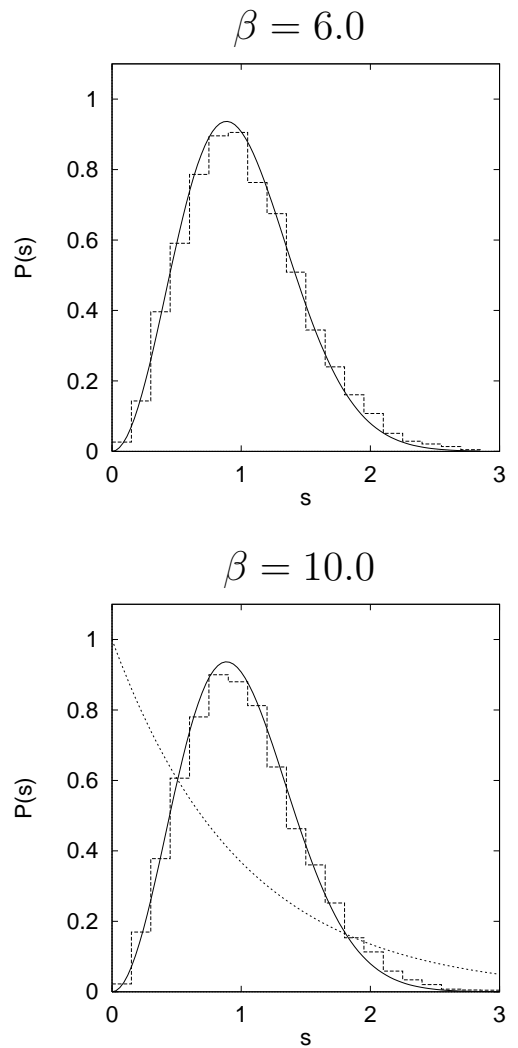

Fig. 2. The nearest-neighbor spacing distribution $P(s)$ averaged over 10 independent configurations (except for $\beta=2.8$ where all single configurations are plotted to give an indication of the deviations) on a $6^{3} \times 4$ lattice in pure $\mathrm{SU}(3)$ (histograms) compared with the random-matrix result (solid lines). For comparison, the Poisson distribution $P(s)=e^{-s}$ is plotted for $\beta=10.0$ (dotted line). There are no changes in $P(s)$ across the deconfinement phase transition.

\section{Confinement $\beta=5.2 \quad$ Deconfinement $\beta=5.4$}

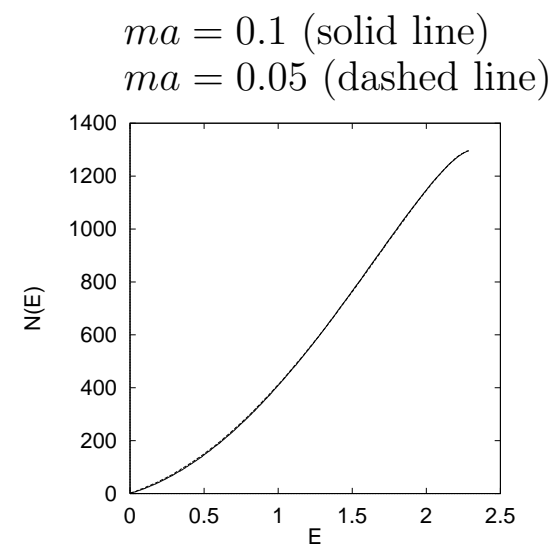

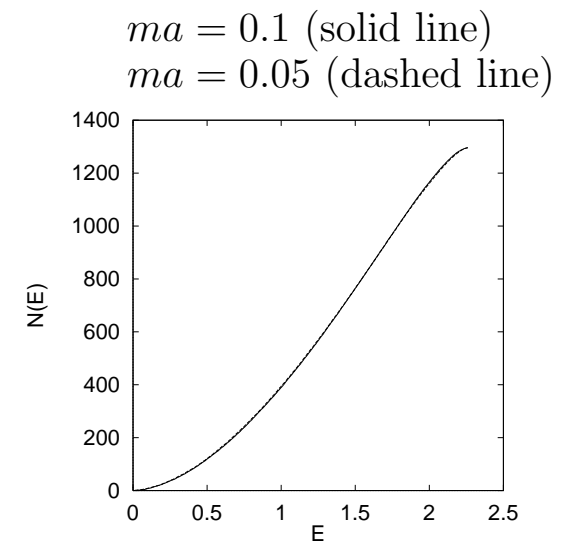

Fig. 3. The staircase function $N(E)$ for typical configurations on a $6^{3} \times 4$ lattice in full QCD with different quark masses. 
Confinement $\beta=5.2$
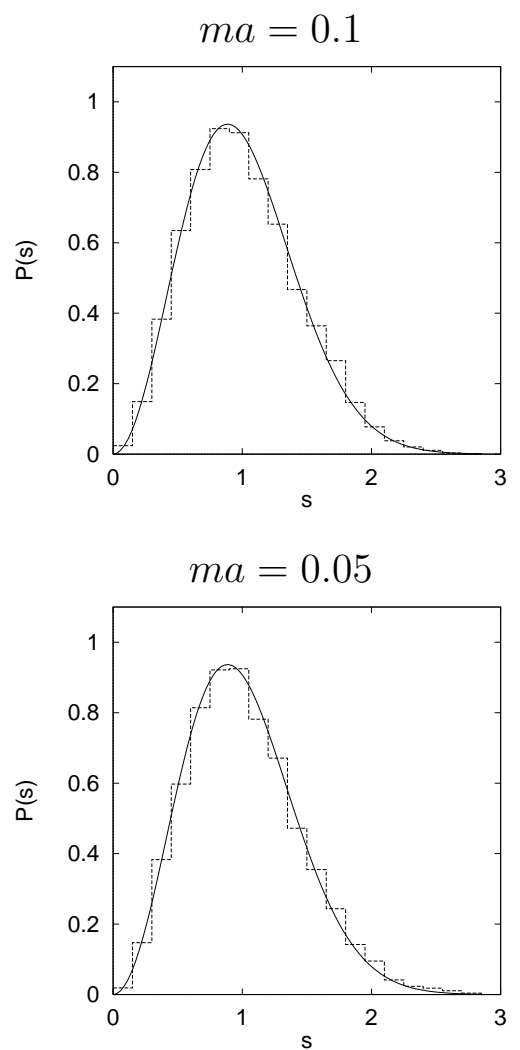

Deconfinement $\beta=5.4$
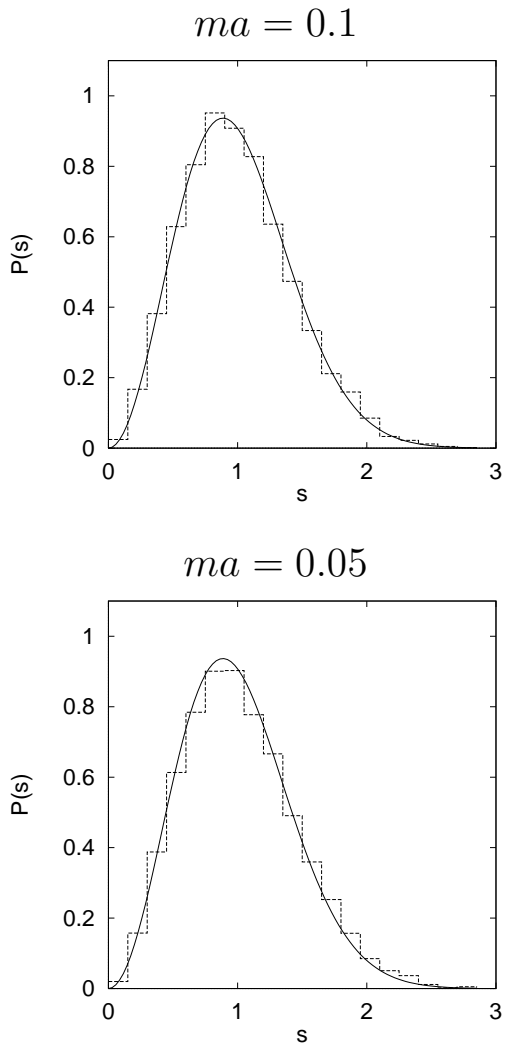

Fig. 4. The nearest-neighbor spacing distribution $P(s)$ averaged over 10 independent configurations on a $6^{3} \times 4$ lattice in full QCD (histograms) compared with the random-matrix result (solid lines). Again, there are no changes in $P(s)$ across the deconfinement phase transition.

tion. While it may be interesting to search for a Wigner to Poisson transition on such asymmetric lattices, it is unlikely that such a transition will coincide with the deconfinement phase transition.

We do not believe that the absence of a signature for a transition from Wigner to Poisson behavior at the deconfinement phase transition is due to the finite lattice size. Even for the small lattice size we used, the agreement of $P(s)$ with the RMT curve is nearly perfect. This leads us to believe that we should have seen some sign of a transition if it existed in the thermodynamic limit.

\section{Conclusions}

We have searched for a transition in the nearest-neighbor spacing distribution $P(s)$ from Wigner to Poisson behavior across the deconfinement phase transition of pure gluonic and of full QCD. Such a transition exists, e.g., at 
the localization transition in disordered mesoscopic systems [5]. In a YangMills-Higgs system a smooth transition along a Brody distribution was seen [7]. We observed no signature of a transition in our data for the lattice Dirac matrix, neither for pure SU(3) nor for full QCD. The data agree with the RMT result in both the confinement and the deconfinement phase except for extremely large values of $\beta$ where the eigenvalues are known analytically. Our analysis of full QCD with two different quark masses showed no influence on the nearest-neighbor spacing distribution.

Our findings are consistent with earlier studies of the chaotic dynamics in classical lattice gauge theories which have shown that lattice gauge theories are chaotic as classical Hamiltonian dynamical systems [14]. Also, it was found recently that the leading Lyapunov exponents of SU(2) Yang-Mills field configurations indicate that configurations in the deconfinement phase are still chaotic, although less chaotic than in the strong coupling phase at finite temperature [15].

In hindsight, our results are not totally unexpected. Temporal monopole currents survive the deconfinement phase transition leading to confinement of spatial Wilson loops. Thus, even in the deconfinement phase, the gauge fields retain a certain degree of randomness. It was shown that the relevant parameter that drives the transition from Poisson regularity to chaos is of the order of the mean level spacing $\Delta$ of the system [16,9]. One could search for a formal separation of the Dirac operator into a regular and a chaotic contribution,

$\not D=\not D_{\text {reg }}+(\alpha / \Delta) \not D_{\text {chaotic }}$. Then, a chaotic perturbation of size $\alpha \gtrsim \Delta$ will generate a Wigner distribution. In future investigations, it would be interesting to try to disentangle the corresponding contributions to the Dirac matrix.

\section{Acknowledgments}

This work was supported in part by FWF project P10468-PHY and by DFG grant We 655/11-2. We thank T.S. Biró, M. Göckeler, T. Guhr, E.-M. Ilgenfritz, M.I. Polikarpov, P. Rakow, A. Schäfer, and J.J.M. Verbaarschot for helpful discussions.

\section{References}

[1] T. Banks and A. Casher, Nucl. Phys. B 169 (1980) 103.

[2] M.A. Halasz and J.J.M. Verbaarschot, Phys. Rev. Lett. 74 (1995) 3920; M.A. Halasz, T. Kalkreuter, and J.J.M. Verbaarschot, Nucl. Phys. B (Proc. Suppl.) 53 (1997) 266. 
[3] O. Bohigas, M.-J. Giannoni, and C. Schmit, Phys. Rev. Lett. 52 (1984) 1.

[4] Reviews on the subject of quantum chaos are: M.C. Gutzwiller, Chaos in Classical and Quantum Mechanics (Springer, New York, 1990); Proceedings of the LII Session of the Les Houches School, Chaos and Quantum Physics, Eds. M.-J. Giannoni and A. Voros (North-Holland, Amsterdam, 1991); Quantum Chaos: Between Order and Disorder, Eds. G. Casati and B.V. Chirikov (Cambridge Univ. Press, Cambridge, 1995).

[5] B.L. Al'tshuler, I.Kh. Zharekeshev, S.A. Kotochigova, and B.I. Shklovskiǔ, Zh. Eksp. Teor. Fiz. 94 (1988) 343 [Sov. Phys. JETP 67 (1988) 625]; B.I. Shklovskiǔ, B. Shapiro, B.R. Sears, P. Lambrianides, and H.B. Shore, Phys. Rev. B 47 (1993) 11487.

[6] H. Markum, R. Pullirsch, K. Rabitsch, and T. Wettig, Nucl. Phys. B (Proc. Suppl.) 63A-C (1998) 832.

[7] L. Salasnich, Mod. Phys. Lett. A 12 (1997) 1473.

[8] L. Casetti, R. Gatto, and M. Modugno, Phys. Rev. E57 (1998) R1223.

[9] T. Guhr, Phys. Rev. Lett. 76 (1996) 2258; Ann. Phys. (NY) 250 (1996) 145.

[10] J.J.M. Verbaarschot, Phys. Rev. Lett. 72 (1994) 2531.

[11] D. Fox and P.B. Kahn, Phys. Rev. 134 (1964) B1151; T. Nagao and M. Wadati, J. Phys. Soc. Jpn. 60 (1991) 3298; 61 (1992) 78, 1910.

[12] M.L. Mehta, Random Matrices, 2nd ed. (Academic Press, San Diego, 1991).

[13] J.J.M. Verbaarschot, private communication.

[14] T.S. Biró, C. Gong, B. Müller, and A. Trayanov, Int. J. Mod. Phys. C5 (1994) 113.

[15] T.S. Biró, M. Feurstein, and H. Markum, APH Heavy Ion Physics 7 (1998) 235.

[16] A. Pandey, Ann. Phys. (NY) 134 (1981) 110. 